\title{
Finding a Common Weight Vector of Data Envelopment Analysis Based upon Bargaining Game
}

\author{
Manabu Sugiyama ${ }^{1} \&$ Toshiyuki Sueyoshi ${ }^{2}$ \\ ${ }^{1}$ Faculty of Social and Information Studies, Gunma University, Maebashi-City, Gunma, Japan \\ ${ }^{2}$ Department of Management, New Mexico Institute of Mining \& Technology, Socorro, NM, USA \\ Correspondence: Manabu Sugiyama, Faculty of Social and Information Studies, Gunma University, 4-2 Aramaki-machi, \\ Maebashi-City, Gunma 371-8510, Japan. Tel: 81-27-220-7522. E-mail: sugi@ si.gunma-u.ac.jp
}

Received: October 20, 2013 Accepted: November 6, 2013 Available online: November 11, 2013

doi:10.11114/set.v1i1.277

URL: http://dx.doi.org/10.11114/set.v1i1.277

\begin{abstract}
Data Envelopment Analysis (DEA) is a mathematical programming method for measuring the relative efficiency of Decision Making Units (DMUs) by evaluating their outputs and inputs. In the history of DEA, the cross-efficiency of $j$ th DMU is widely used as an efficiency measure of a given $\mathrm{DMU}_{o}$ among researchers. The approach always utilizes weights related to inputs and outputs in the assessment. Unfortunately, the weights are not always uniquely determined in the cross-efficiency measurement because DEA always suffers from an occurrence of multiple solutions, so indicating an occurrence of multiple weights. To overcome such a difficulty, this paper proposes a new approach for determining a common weight vector of DEA based on bargaining game.
\end{abstract}

Keywords: Bargaining Game, Kalai-Smorodinsky Bargaining Solution, Data Envelopment Analysis, Common Weight Vector

\section{Introduction}

A large number of studies on Data Envelopment Analysis (DEA) have developed after DEA was first proposed by Charnes, Cooper and Rhodes (1978), as confirmed by Glover and Sueyoshi (2009). DEA is a mathematical programming approach to assess relative efficiencies within a group of Decision Making Units (DMUs). An important result of such an analysis is a set of virtual multipliers, or weights, accorded to production factors (i.e., inputs or outputs). The set of weights are often different for each of the participating DMUs.

Sexton, Silkman and Hogan (1986) have defined the cross-efficiency of $j$ th $\mathrm{DMU}\left(\mathrm{DMU}_{j}\right)$ as a measure of $\mathrm{DMU}_{o}$ that is the ratio of weighted outputs to weighted inputs obtained when we use both input and output levels of $\mathrm{DMU}_{j}$. There were several research efforts (e.g. Kao \& Hung, 2005, Sugiyama \& Yamada, 2001) that applied the cross-efficiency. Moreover, there were other articles (e.g. Kao \& Hung, 2005, Boussofiane \& Dyson, 1991, Cook, Kress \& Seiford, 1992, and Roll, Cook \& Golany, 1991) that discussed about a use of common weights. However, it is widely known that the weights are not always uniquely determined. Therefore, the cross-efficiency method is not uniquely determined in DEA.

The DEA measurement process regarding efficiency of each DMU can be considered as playing a bargaining game (Peters, 1992, and Thomson, 1994). In some cases, the measurement of relative efficiency by using a scheme of the bargaining game is useful for group decision making. Thus, this study proposes a new approach for determining a common weight vector based on the bargaining game. Furthermore, this research uses an example on Japanese electric power industries in order to document the practicality of the proposed approach.

The remainder of this paper is organized as follows: Section 2 introduces a basic DEA model for making the proposed analysis that incorporates cross-efficiency and its related total efficiency measures. Section 3 defines a feasible set from the bargaining game for DEA. The section also proposes the calculation on Kalai-Smorodinsky bargaining solution by a feasible set. Section 4 applies the proposed approach to measure the productivity analysis of Japanese electric power industry. Section 5 summarizes conclusions and future extensions.

\section{DEA Model and the Cross-efficiency}

There are various descriptions about DEA. To uniform symbols and expressions, this study follows a description of Cooper, Seiford and Tone (2006). 


\subsection{DEA Model}

DEA presupposes total " $n$ " DMUs as research objects of its analysis. It is also assumed that each $\operatorname{DMU}_{j}(j=1,2, \ldots, n)$ has common inputs and outputs which contain $m$ inputs $x_{i j}>0(i=1,2, \ldots, m)$ and $s$ outputs $y_{r j}>0(r=1,2, \ldots, s)$. Input-oriented radial model under constant Returns to Scale (RTS), which is a basic DEA model on $\operatorname{DMU}_{o}(o=1,2, \ldots, n)$ to be examined, has the following mathematical formulation:

$$
\begin{array}{cc}
\text { min. } \theta_{o}-\varepsilon\left(\sum_{i=1}^{m} s_{i o}^{-}+\sum_{r=1}^{s} s_{r o}^{+}\right), & \\
\text {subject to } \theta_{o} x_{i o}-\sum_{j=1}^{n} x_{i j} \lambda_{j o}-s_{i o}^{-}=0 & (i=1,2, \ldots, m), \\
\sum_{j=1}^{n} y_{r j} \lambda_{j o}-s_{r o}^{+}=y_{r o} & (r=1,2, \ldots, s), \\
\lambda_{j o} \geq 0 & (j=1,2, \ldots, n), \\
s_{r o}^{+} \geq 0 & (r=1,2, \ldots, s), \\
s_{i o}^{-} \geq 0 & (i=1,2, \ldots, m) .
\end{array}
$$

The dual form of Model (1) are expressed by

$$
\begin{aligned}
& \max . \xi_{o}=\sum_{r=1}^{s} u_{r o} y_{r o}, \\
& \text { subject to } \sum_{i=1}^{m} v_{i o} x_{i o}=1 \text {, } \\
& -\sum_{i=1}^{m} v_{i o} x_{i j}+\sum_{r=1}^{s} u_{r o} y_{r j} \leq 0 \quad(j=1,2, \ldots, n), \\
& u_{r o} \geq \varepsilon \quad(r=1,2, \ldots, s), \\
& v_{i o} \geq \varepsilon \quad(i=1,2, \ldots, m) .
\end{aligned}
$$

The $\mathrm{DMU}_{o}$ has an efficiency measure $\theta_{o}$ that is obtained by a relative comparison with total $n$ of DMUs' production activities. The optimal value $\theta_{o}^{*}$ obtained from Model (1) is often called "technical efficiency" in production economics. The status of DEA-efficiency needs both $\theta_{o}^{*}=1$ and all slack variables are zero, i.e., $s_{r o}^{*^{*}}=0$ for all $r \in\{1,2, \ldots, s\}$ and $s_{i o}^{-*}=0$ for all $i \in\{1,2, \ldots, m\}$. If $\theta_{o}^{*}=1$ and at least one or more slack variables are $s_{r o}^{+*}>0$ for some $r \in\{1,2, \ldots, s\}$ and $s_{i o}^{-*}>0$ for some $i \in\{1,2, \ldots, m\}$ or $\theta_{o}^{*}<1, \mathrm{DMU}_{o}$ is defined as inefficient. Hence, Model (1) can be usually solved by two steps of optimization without providing a specific value to $\varepsilon$, which is a non-Archimedean infinitesimal (Cooper et al., 2006). This study extends the discussion by using this radial model under constant RTS. The radial model is expressed as:

$$
\begin{array}{cc}
\max . \eta_{o}=\frac{\sum_{r=1}^{s} u_{r o} y_{r o}}{\sum_{i=1}^{m} v_{i o} x_{i o}}, & \\
\text { subject to } & \frac{\sum_{r=1}^{s} u_{r o} y_{r j}}{\sum_{i=1}^{m} v_{i o} x_{i j}} \leq 1 \quad(j=1,2, \ldots, n), \\
u_{r o} \geq \varepsilon & (r=1,2, \ldots, s), \\
v_{i o} \geq \varepsilon & (i=1,2, \ldots, m) .
\end{array}
$$

Here, let $u_{r o}^{*}$ be an optimal value of $u_{r o}$, and, let $v_{i o}^{*}$ be an optimal value of $v_{i o}$ in the Model (2). This study refers to these $u_{r o}^{*}$ and $v_{i o}^{*}$ as DEA-solutions.

\subsection{The Cross-efficiency and the Accommodated Total Efficiency}

The cross-efficiency was first proposed by Sexton et al. (1986). They have defined the cross-efficiency of $\mathrm{DMU}_{j}$ as measured by $\mathrm{DMU}_{o}$ as the ratio of weighted outputs to weighted inputs obtained when we use the input and output levels of $\mathrm{DMU}_{j}$ along with these weights derived for $\mathrm{DMU}_{o}$, as discussed previously. Mathematically, the 
cross-efficiency is the ratio of the sums on the left side of constraint $j$ in Model (3) for $\mathrm{DMU}_{o}$ :

$$
E_{o j}=\frac{\sum_{r=1}^{s} u_{r o}^{*} y_{r j}}{\sum_{i=1}^{m} v_{i o}^{*} x_{i j}} \quad(o=1,2, \ldots, n ; j=1,2, \ldots, n) .
$$

The cross-efficiencies are simply the ratios in the constraints of Model (3). The cross-efficiencies are easily summarized by an $n \times n$ matrix, whose $(o, j)$ component is $E_{o j}$. Sexton et al. (1986) called it as cross-efficiency matrix. The conventional efficiency measures exist on the diagonal of the cross-efficiency matrix.

By examining the row $o$ of the cross-efficiency matrix, this study can identify how $\mathrm{DMU}_{o}$ rates each of the other DMUs, that is, how efficient each of the other DMUs is when an optimal weights generated by $\mathrm{DMU}_{o}$ are used for its measurement. The mean efficiency in the row $o$ (including the diagonal) is called $\operatorname{EROW}(o)\left(=\frac{1}{n} \sum_{j=1}^{n} E_{o j}\right)$. The measure indicates the average efficiency of all DMUs according to $\mathrm{DMU}_{o}$. In a similar manner, this study can examine the column $j$ of the cross-efficiency matrix to identify how $\mathrm{DMU}_{j}$ is rated by each of the other DMUs when it is evaluated by means of the optimal weights that they are generated. The mean efficiency in the column $j$ (including the diagonal) is referred to as $\operatorname{ECOL}(j)\left(=\frac{1}{n} \sum_{o=1}^{n} E_{o j}\right)$. The measure indicates the average efficiency of $\mathrm{DMU}_{j}$ according to all other DMUs. They can compute the average of all the cross-efficiency values, or EBAR. However, there may be no common weights of the cross-efficiency because DEA always suffers from an occurrence of multiple solutions.

The accommodated total efficiency was proposed by Sugiyama and Yamada (2001). They showed that the accommodated total efficiency is a general form for the cross-efficiency. The accommodated total efficiencies of DMUs are calculated from the following three steps. (a) In the first step, they evaluate the relative efficiencies of DMUs as group members. (b) In the second step, the mutual evaluation information of DMUs can be defined and calculated by using their weights. They indicate the mutual evaluation information by a form of matrix which they called it as "Accommodation Efficiency Matrix." It is widely known that the weights are not always uniquely determined. The mutual evaluation information is not uniquely determined. Here, they have proposed a method for determining the weights uniquely by minimizing square of the weights differences. (c) In the third step, they calculate the accommodated total efficiency which is the group efficiencies of DMUs by using the maximum eigenvalue of "Accommodation Efficiency Matrix."

\section{A Common Weight Vector by the Bargaining Game Approach}

This section defines some feasible sets of bargaining game on DEA and proposes the Kalai-Smorodinsky bargaining solution by using those feasible sets. There are various descriptions about the bargaining game, and many articles. See, for example, Peters (1992) and Thomson (1994). The study of DEA with the Game theory can be found in Banker, Charnes, Cooper and Clarke (1989), Semple (1996), Hao, Wei and Yan (2000), Nakabayashi and Tone (2006), and etc. On the other hand, Du, Liang, Chen, Cook and Zhu (2011) described DEA on the bargaining game. A Nash bargaining game has also been proposed for measuring the performance of a two-stage network DEA system.

\subsection{The Bargaining Game}

By $N \equiv\{1,2, \ldots n\}$, this study denotes the set of players. The bargaining game (Peters, 1992, and Thomson, 1994) is defined by a pair of $(S, \boldsymbol{d})$. The players in $N$ try to reach a unanimous agreement on some outcome $\boldsymbol{\eta} \in S$, yielding utility $\eta_{k}$ for player $k$. If they fail, the disagreement outcome or disagreement point $\boldsymbol{d}$ occurs in the game. The set $S$ is referred to as a feasible set of the bargaining game. The set $S$ needs to be convex, bounded and closed. There is at least one point of $S$ strictly dominating $\boldsymbol{d}$.

This study chooses one of the bargaining solutions by applying an axiomatic approach. In the axiomatic approach, the typical solution on the bargaining game is the Nash bargaining solution (Thomson, 1994). The Nash bargaining solution is a single solution on the feasible set $S$ satisfying Pareto-optimality, symmetry, scale invariance and independence of irrelevant alternatives (IIA). Here, a solution of the proposed bargaining game on DEA satisfies Pareto-optimality, symmetry and scale invariance. However, the solution of the bargaining game on DEA may not satisfy independence of irrelevant alternatives (IIA). Therefore, the Nash bargaining solution is not appropriate. The rationale is because a DEA-efficiency score is a relative evaluation score.

Meanwhile, a DEA solution of the proposed bargaining game fully satisfies individual monotonicity. The Kalai-Smorodinsky bargaining solution (Thomson, 1994) is the only solution on a feasible set $S$ satisfying Pareto-optimality, symmetry, scale invariance and individual monotonicity. Consequently, this study selects the Kalai-Smorodinsky bargaining solution.

These axioms are described in research efforts (i.e., Peters, 1992, and Thomson, 1994). The definition of the Kalai-Smorodinsky bargaining solution (Thomson, 1994) is specified as follows. 
[The Kalai-Smorodinsky bargaining solution] $K(S)$ :

$K(S)$ is the maximal point of $S$ on a segment connecting the origin to $a(S)$, the ideal point of $S$, defined by $a_{k}(S) \equiv \max \left\{\eta_{k} \mid \boldsymbol{\eta} \in S\right\}$ for all $k$.

[In another definition, $K(S, \boldsymbol{d})$ is the maximal point of $S$ on a segment connecting $\boldsymbol{d}$ to $a(S, \boldsymbol{d})$ where $a_{k}(S, \boldsymbol{d}) \equiv \max \left\{\eta_{k} \mid \boldsymbol{\eta} \in S, \boldsymbol{\eta} \geq \boldsymbol{d}\right\}$ for all $k$.]

\subsection{The Feasible Set $S$ of the Bargaining Game on DEA}

Let the players be DMUs. Consequently, the number of the players is $n$. In addition, game situations assume the bargaining game. Then, this study generalizes a feasible set of bargaining game on DEA. The feasible set in a correlated pure strategy is expressed as follows:

$$
S^{\mathrm{P}}=\left\{\boldsymbol{\eta} \in \mathrm{R}^{n} \mid \begin{array}{ll}
\eta_{j}=\frac{\sum_{r=1}^{s} u_{r} y_{r j}}{\sum_{i=1}^{m} v_{i} x_{i j}} & (j \in N), \\
\frac{\sum_{r=1}^{s} u_{r} y_{r j}}{\sum_{i=1}^{m} v_{i} x_{i j}} & (j \in N), \\
u_{r} \geq 0 & (r=1,2, \ldots, s), \\
v_{i} \geq 0 & (i=1,2, \ldots, m)
\end{array}\right\} .
$$

The feasible set in correlated pure strategy $S^{\mathrm{P}}$ is the feasible set of a common weight vector of DEA. Moreover, the comprehensive hull of $S^{\mathrm{P}}$ is expressed as follows:

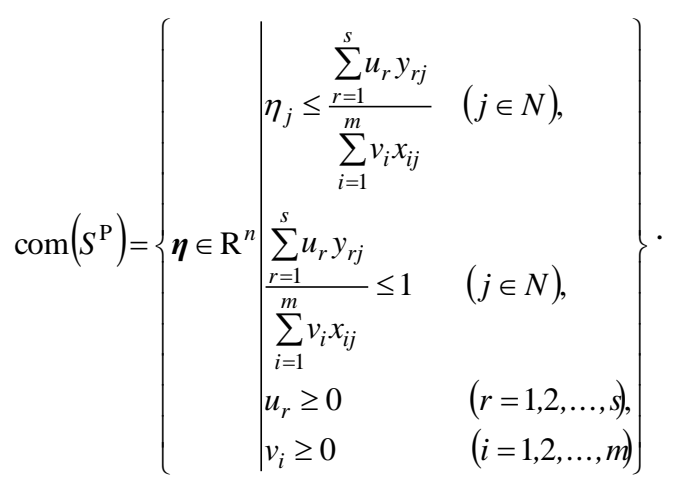

Here, it is expected that the feasible set in correlated pure strategy $S^{\mathrm{P}}$ is a convex set. However, it is difficult to prove whether $S^{\mathrm{P}}$ is a convex set because $S^{\mathrm{P}}$ includes the fractional equations. Let the input and output data for $\mathrm{DMU}_{j}$ be $\boldsymbol{x}_{j}=\left(x_{1 j}, x_{2 j}, \ldots, x_{m j}\right)^{\mathrm{T}}$ and $\boldsymbol{y}_{j}=\left(y_{1 j}, y_{2 j}, \ldots, y_{m j}\right)^{\mathrm{T}}$, respectively. The symbol " $\mathrm{T}$ " denotes vector transpose. In addition, let the weight vector be $\boldsymbol{u}=\left(u_{1}, u_{2}, \ldots, \mu_{s}\right)$ and $\boldsymbol{v}=\left(v_{1}, v_{2}, \ldots, v_{m}\right)$. Therefore, this study sets Model (6) by relaxing the equality constraints $\eta_{j}=\boldsymbol{u} \boldsymbol{y}_{j} / \boldsymbol{v} \boldsymbol{x}_{j}$ into inequality ones. This relaxation of $\operatorname{com}\left(S^{\mathrm{P}}\right)$ is naturally accepted in the bargaining game. Then, this study calculates the Kalai-Smorodinsky bargaining solution by these feasible sets in the proceeding section. By comparing results obtained by the Kalai-Smorodinsky bargaining solution that obtains from the two feasible sets, it is possible to confirm that these feasible solutions are convex sets near the Kalai-Smorodinsky bargaining solution.

\subsection{The Computational Mode for Bargaining Solution}

Let the ideal point of $S$ be each DMU's efficiency score $\theta_{j}^{*}$. The proposed bargaining game can establish various kinds of points as the disagreement point $\boldsymbol{d}$. Therefore, this study sets the disagreement point $\boldsymbol{d}$ as the origin. However, this study cannot accept the bargaining solution from the origin. Because the DEA-solutions become $\boldsymbol{u}^{*}=\boldsymbol{0}$ or $\boldsymbol{v}^{*}=\boldsymbol{0}$. The weight of the DEA problem (3) is a non-zero vector. Since $\boldsymbol{\theta}=\boldsymbol{\eta} \in S^{\mathrm{P}}$ and $\boldsymbol{u}=\boldsymbol{0}$ are equivalent, a Kalai-Smorodinsky bargaining solution $\boldsymbol{\eta}$ such as a common weight vector of DEA is a non-zero vector. In addition, since $\boldsymbol{y}_{j}>\boldsymbol{0}$ for all $j \in N, \boldsymbol{u} \geq \boldsymbol{0}$ and $\boldsymbol{u} \neq \boldsymbol{0}$, and $\boldsymbol{\eta}>\boldsymbol{0}$ are equivalent. Therefore, this study refers to as a Kalai-Smorodinsky bargaining solution $\boldsymbol{\eta} \neq \boldsymbol{0}$ as a positive Kalai-Smorodinsky bargaining solution. A Kalai-Smorodinsky bargaining solution corresponds to a desirable common DEA weights is a positive Kalai-Smorodinsky bargaining solution. 
Since $a\left(S^{\mathrm{P}}\right)=\left(\theta_{1}^{*}, \theta_{2}^{*}, \ldots, \theta_{n}^{*}\right)$ and a line segment between the origin $\boldsymbol{O}$ and $a\left(S^{\mathrm{P}}\right)$ is $\left\{\boldsymbol{\eta} a\left(S^{\mathrm{P}}\right) 0 \leq \eta_{j} \leq 1, j \in N\right\}$, finding a positive Kalai-Smorodinsky bargaining solution $K\left(S^{\mathrm{P}}\right)$ of $\left(S^{\mathrm{P}}, 0\right)$ is the same of solving the positive optimal value $\eta_{j}^{*}$ of

$$
\begin{array}{ll}
\max . & \sum_{j=1}^{n} \theta_{j}^{*} \eta_{j}, \\
\text { subject to } & \frac{\sum_{r=1}^{s} u_{r} y_{r j}}{\sum_{i=1}^{m} v_{i} x_{i j}}=\eta_{j} \quad(j \in N), \\
& \frac{\sum_{r=1}^{s} u_{r} y_{r j}}{\sum_{i=1}^{m} v_{i} x_{i j}} \leq 1 \quad(j \in N), \\
& 1 \geq \eta_{j} \geq 0 \quad(j \in N), \\
& u_{r} \geq 0 \quad(r=1,2, \ldots, s), \\
v_{i} \geq 0 \quad(i=1,2, \ldots, m) .
\end{array}
$$

The maximization of Model (7) is an unbounded problem. Thus, the maximization problem form, modified by adding the equation $v x_{j} \geq 1$, becomes:

$$
\begin{array}{cl}
\operatorname{max.} & \sum_{j=1}^{n}\left(\theta_{j}^{*} \frac{\sum_{r=1}^{s} u_{r} y_{r j}}{\sum_{i=1}^{m} v_{i} x_{i j}}\right), \\
\text { subject to } & \sum_{i=1}^{m} v_{i} x_{i j} \geq 1 \\
& -\sum_{i=1}^{m} v_{i} x_{i j}+\sum_{r=1}^{s} u_{r} y_{r j} \leq 0 \quad(j \in N), \\
& u_{r} \geq 0 \quad \quad(r=1,2, \ldots, s), \\
v_{i} \geq 0 & (i=1,2, \ldots, m) .
\end{array}
$$

If $\operatorname{com}\left(S^{\mathrm{P}}\right)$ is a feasible set of the bargaining game, then finding a Kalai-Smorodinsky bargaining solution $K\left(\operatorname{com}\left(S^{\mathrm{P}}\right)\right)$ of $\left(\operatorname{com}\left(S^{\mathrm{P}}\right), 0\right)$ is the same of solving the positive optimal value $\eta_{j}^{*}$ of

$$
\begin{array}{ll}
\max . & \sum_{j=1}^{n} \theta_{j}^{*} \eta_{j}, \\
\text { subject to } & \frac{\sum_{r=1}^{s} u_{r} y_{r j}}{\sum_{i=1}^{m} v_{i} x_{i j}} \geq \eta_{j} \quad(j \in N), \\
& \frac{\sum_{r=1}^{s} u_{r} y_{r j}}{\sum_{i=1}^{m} v_{i} x_{i j}} \leq 1 \quad(j \in N), \\
& 1 \geq \eta_{j} \geq 0, \quad(j \in N), \\
& u_{r} \geq 0 \quad(r=1,2, \ldots, s), \\
& v_{i} \geq 0 \quad(i=1,2, \ldots, m) .
\end{array}
$$

The maximization of Model (9) is an unbounded problem, as well. Thus, the maximization problem by adding the equation $\boldsymbol{v} \boldsymbol{x}_{j} \geq 1$ becomes as follows: 


$$
\begin{aligned}
& \max . \quad \sum_{j=1}^{n} \theta_{j}^{*} \eta_{j}, \\
& \text { subject to } \sum_{i=1}^{m} v_{i} x_{i j} \geq 1 \quad(j \in N) \text {, } \\
& -\eta_{j} \sum_{i=1}^{m} v_{i} x_{i j}+\sum_{r=1}^{s} u_{r} y_{r j} \geq 0 \quad(j \in N), \\
& -\sum_{i=1}^{m} v_{i} x_{i j}+\sum_{r=1}^{s} u_{r} y_{r j} \leq 0 \quad(j \in N), \\
& 1 \geq \eta_{j} \geq 0, \quad(j \in N), \\
& u_{r} \geq 0 \quad(r=1,2, \ldots, s) \text {, } \\
& v_{i} \geq 0 \quad(i=1,2, \ldots, m) .
\end{aligned}
$$

That is, $K\left(\operatorname{com}\left(S^{\mathrm{P}}\right)\right)=\boldsymbol{\eta}^{*} a\left(\operatorname{com}\left(S^{\mathrm{P}}\right)\right)$. Here, this study can set the ideal point of $S$ be an each DMU's efficiency score. Furthermore, it is possible to set the disagreement point $\boldsymbol{d}$ as the origin.

\section{Numerical Example}

This section documents the productivity analysis of Japanese electric power industry by applying the proposed approach. This example was given in Sugiyama and Yamada (2001).

\subsection{Data}

The subjects of analysis are nine electric power companies in Japan. This study utilizes the management indexes, given below, as input/output data of each company in the fiscal year 1991. The data source is "Hand Book of Electric Power Industry '91" (Statistics Committee of Electric Utilities Association (Ed.), 1992), in the form of Table 1.

\section{[Input/Output Items]}

Inputs: $\quad x_{1 j}$ "Number of Employees", $x_{2 j}$ "Maximum Generation Capacity" and $x_{3 j}$ "Total Assets".

Outputs : $y_{1 j}$ "Electricity Sales" and $y_{2 j}$ "Number of Customers".

Table 1. Observed Inputs and Outputs

\begin{tabular}{lrrrrr}
\hline & $\begin{array}{c}\text { Number of } \\
\text { Employees } \\
x_{1 j}\end{array}$ & $\begin{array}{c}\text { Maximum } \\
\text { Gen. Cap. } \\
x_{2 j}\end{array}$ & $\begin{array}{c}\text { Total } \\
\text { Assets } \\
x_{3 j}\end{array}$ & $\begin{array}{c}\text { Electricity } \\
\text { Sales }\end{array}$ & \multicolumn{2}{c}{$\begin{array}{c}\text { Number of } \\
\text { Customers }\end{array}$} \\
\hline Hokkaido & 6,457 & 5,315 & $1,320,938$ & 21,389 & \multicolumn{1}{c}{$y_{2 j}$} \\
Tohoku & 13,557 & 10,150 & $2,657,112$ & 55,227 & 6,256 \\
Tokyo & 40,063 & 46,905 & $11,627,131$ & 227,631 & 23,221 \\
Chubu & 20,285 & 22,799 & $4,896,313$ & 103,140 & 8,711 \\
Hokuriku & 5,338 & 4,453 & $1,252,893$ & 21,711 & 1,712 \\
Kansai & 25,166 & 33,158 & $5,931,094$ & 122,749 & 11,331 \\
Chugoku & 10,898 & 9,433 & $2,148,717$ & 44,498 & 4,578 \\
Shikoku & 6,603 & 5,423 & $1,214,685$ & 20,548 & 2,490 \\
Kyushu & 13,669 & 14,063 & $3,305,687$ & 57,272 & 7,007 \\
\hline Scale & $\times 10^{0}$ & $\times 10^{3}(\mathrm{~kW})$ & $\times 10^{6}(\mathrm{YEN})$ & $\times 10^{6}(\mathrm{kWh})$ & $\times 10^{3}$ \\
\hline
\end{tabular}

Table 2. Modified Input/Output Data by Average

\begin{tabular}{lccccc}
\hline & $\begin{array}{c}\text { Number of } \\
\text { Employees } \\
x_{1 j}\end{array}$ & $\begin{array}{c}\text { Maximum } \\
\text { Gen. Cap. } \\
x_{2 j}\end{array}$ & $\begin{array}{c}\text { Total } \\
\text { Assets }\end{array}$ & $\begin{array}{c}\text { Electricity } \\
\text { Sales }\end{array}$ & $\begin{array}{c}\text { Number of } \\
\text { Customers } \\
x_{3 j}\end{array}$ \\
\hline Hokkaido & 0.4091 & 0.3153 & 0.3461 & 0.2855 & 0.4262 \\
Tohoku & 0.8590 & 0.6022 & 0.6961 & 0.7373 & 0.8437 \\
Tokyo & 2.5386 & 2.7828 & 3.0460 & 3.0388 & 3.0398 \\
Chubu & 1.2853 & 1.3526 & 1.2827 & 1.3769 & 1.1403 \\
Hokuriku & 0.3382 & 0.2642 & 0.3282 & 0.2898 & 0.2241 \\
Kansai & 1.5946 & 1.9672 & 1.5538 & 1.6387 & 1.4833 \\
Chugoku & 0.6905 & 0.5596 & 0.5629 & 0.5940 & 0.5993 \\
Shikoku & 0.4184 & 0.3217 & 0.3182 & 0.2743 & 0.3260 \\
Kyushu & 0.8661 & 0.8343 & 0.8660 & 0.7646 & 0.9173 \\
\hline
\end{tabular}




\subsection{Analysis and Evaluation}

First, DEA is appiled on the nine electric power companies as DMUs. Table 3 summarizes the computational results obtained from the proposed analysis, i.e., DEA-efficiency scores $\theta^{*}$ and its reference set, for each DMU. As a result, Hokkaido, Tohoku, Tokyo and Chubu are determined DEA-efficient. In contrast, Hokuriku, Kansai, Chugoku, Shikoku and Kyushu are determined DEA-inefficient.

Table 3. DEA-efficiency of Electric Power Companies

\begin{tabular}{lcc} 
& $\begin{array}{c}\text { DEA-efficiency } \\
\theta_{o}^{*}\end{array}$ & reference set \\
\hline Hokkaido & 1.0000 & --- \\
Tohoku & 1.0000 & --- \\
Tokyo & 1.0000 & --- \\
Chubu & 1.0000 & --- \\
Hokuriku & 0.9245 & Tohoku, Tokyo \\
Kansai & 0.9928 & Tohoku, Tokyo, Chubu \\
Chugoku & 0.9906 & Tohoku, Chubu \\
Shikoku & 0.8430 & Hokkaido, Tohoku \\
Kyushu & 0.9560 & Hokkaido, Tohoku, Tokyo \\
\hline
\end{tabular}

Second, the study calculates the efficiency of each electric power company from a DEA common weight vector based on the Kalai-Smorodinsky bargaining solution. As discussed in Section 3.3, let the ideal point of $S$ be an each DMU's efficiency score. The proposed bargaining game can establish various kinds of points as the disagreement point $\boldsymbol{d}$. This study sets the disagreement point $\boldsymbol{d}$ as the origin. To calculate the Kalai-Smorodinsky bargaining solution, this study uses the maximization problems (8) and (10), modeled by each feasible set: $a(S)$ and $\boldsymbol{d}$. Tables 4 and 5 indicate the results on efficiencies for each DMU and a common weight vector obtained from the Kalai-Smorodinsky bargaining solution.

Table 4. The Kalai-Smorodinsky Bargaining Solution of Electric Power Companies

\begin{tabular}{|c|c|c|c|}
\hline & \multicolumn{2}{|c|}{ The Kalai-Smorodinsky Bargaining Solution } & \multirow{2}{*}{$\begin{array}{c}\text { DEA-efficiency } \\
\theta_{o}^{*}\end{array}$} \\
\hline & the feasible set $S^{\mathrm{P}}$ & the feasible set $\operatorname{com}\left(S^{\mathrm{P}}\right)$ & \\
\hline Hokkaido & 0.8411 & 0.8411 & 1.0000 \\
\hline Tohoku & 1.0000 & 1.0000 & 1.0000 \\
\hline Tokyo & 1.0000 & 1.0000 & 1.0000 \\
\hline Chubu & 1.0000 & 1.0000 & 1.0000 \\
\hline Hokuriku & 0.8082 & 0.8082 & 0.9245 \\
\hline Kansai & 0.9928 & 0.9928 & 0.9928 \\
\hline Chugoku & 0.9716 & 0.9716 & 0.9906 \\
\hline Shikoku & 0.8071 & 0.8071 & 0.8430 \\
\hline Kyushu & 0.8863 & 0.8863 & 0.9560 \\
\hline
\end{tabular}

Table 5. A Common Weight Vector

\begin{tabular}{llccccc}
\hline & $\bar{v}_{1}$ & $\bar{v}_{2}$ & $\bar{u}_{1}$ & $\bar{u}_{2}$ & $\bar{u}_{3}$ \\
\hline the feasible set & $S^{\mathrm{P}}$ & 0.6779 & 0.0000 & 2.3778 & 2.3584 & 0.5912 \\
the feasible set & $\operatorname{com}\left(S^{\mathrm{P}}\right)$ & 0.6779 & 0.0000 & 2.3778 & 2.3584 & 0.5912 \\
\hline
\end{tabular}

In Tables 4 and 5 , the results based on the feasible set $S^{\mathrm{P}}$ and the feasible set com $\left(S^{\mathrm{P}}\right)$ are same in each company. Consequently, this study assumes that the Kalai-Smorodinsky bargaining solution, the feasible set $S^{\mathrm{P}}$ and $\operatorname{com}\left(S^{\mathrm{P}}\right)$ are a convex set. Therefore, this study determines that Tohoku, Tokyo and Chubu are efficient. Hokkaido, Hokuriku, Kansai, Chugoku, Shikoku and Kyushu are inefficient. Thus, this study is able to identify that Tohoku, Tokyo and Chubu attain a desirable performance level. On the other hand, Hokkaido, Hokuriku, Kansai, Chugoku, Shikoku and Kyushu do not attain the desirable performance level. In this study, the calculated common weight $\bar{v}_{2}$ score is $\bar{v}_{2}=0.0000$. In other words, the input item $x_{2 j}$ "Maximum Generation Capacity" may not be necessary for measuring the relative efficiencies of DMUs. The details of this analysis will become very important information at selecting the input/output items on DEA. 
Table 6. Efficiency of Nine Electric Power Companies

\begin{tabular}{lccc}
\hline & $\begin{array}{c}\text { The Kalai-Smorodinsky } \\
\text { Bargaining Solution }\end{array}$ & $\begin{array}{c}\text { The Accommodated } \\
\text { Total Efficiency }\end{array}$ & $\begin{array}{c}\text { The Cross-Efficiency } \\
\text { (by the weights } \\
\text { determined uniquely) }\end{array}$ \\
\hline Hokkaido & 0.8411 & 0.9155 & 0.8884 \\
Tohoku & 1.0000 & 1.0000 & 0.9757 \\
Tokyo & 1.0000 & 0.9658 & 0.9419 \\
Chubu & 1.0000 & 0.8643 & 0.8476 \\
Hokuriku & 0.8082 & 0.7244 & 0.7120 \\
Kansai & 0.9928 & 0.8500 & 0.8321 \\
Chugoku & 0.9716 & 0.9018 & 0.8824 \\
Shikoku & 0.8071 & 0.7879 & 0.7689 \\
Kyushu & 0.8863 & 0.8893 & 0.8655 \\
\hline
\end{tabular}

Third, Table 6 indicates the results obtained from the Kalai-Smorodinsky bargaining solution and conventional approaches. The accommodated total efficiencies of each DMU were given in Sugiyama and Yamada (2001), as well. Furthermore, the cross-efficiencies $(\operatorname{ECOL}(j))$ of each DMU were calculated by using the weights determined uniquely in Sugiyama and Yamada (2001). The Kalai-Smorodinsky bargaining solution satisfies Pareto-optimality, symmetry, scale invariance and individual monotonicity, thus the result obtained from the Kalai-Smorodinsky bargaining solution may be more useful for a group decision making than other efficiencies. Therefore, each DMU may be able to accept the solution more easily.

Fourth, Table 7 indicates a common weight vector obtained from each approach. Here, the weights of the accommodated total efficiency were given in Sugiyama and Yamada (2001), as well. There were not the weights satisfying the cross-efficiency in the feasible set of DEA-solutions. Hence, these weights were not calculated in this study.

Table 7. A Common Weight Vector of Each Approach

\begin{tabular}{lccccc}
\hline & $\bar{v}_{1}$ & $\bar{v}_{2}$ & $\bar{u}_{1}$ & $\bar{u}_{2}$ & $\bar{u}_{3}$ \\
\hline The Kalai-Smorodinsky Bargaining Solution & 0.6779 & 0.0000 & 2.3778 & 2.3584 & 0.5912 \\
The Accommodated Total Efficiency & 0.3739 & 0.3199 & 0.6984 & 0.5755 & 0.6824 \\
The Cross-Efficiency & --- & --- & --- & --- & --- \\
\hline
\end{tabular}

\section{Conclusions and Future Extensions}

This research discussed a methodology for determining a common weight vector of DEA based on the Kalai-Smorodinsky bargaining solution. The calculated common weight vector was uniquely determined. This study applied the proposed approach to examine the productivity analysis of Japanese electric power industry. The application indicates the practicality of the proposed approach.

This research is the first effort for applying a scheme of bargaining game to determine a common weight vector of DEA. Furthermore, this paper contributed to the progress of the study of DEA with the Game theory.

In conclusion, it is hoped that this study makes a contribution in DEA. We would like to anxiously wait for future extensions that are originated from this research effort.

\section{Acknowledgement}

This work is supported by JSPS Grant-in-Aid for Scientific Research (C) 23510159.

\section{References}

Banker, R. D., Charnes, A., Cooper, W. W., \& Clarke, R. (1989). Constrained Game Formulations and Interpretations for Data Envelopment Analysis, European Journal of Operational Research, 40, $299-308$. http://dx.doi.org/10.1016/0377-2217(89)90422-0

Boussofiane, A., Dyson, R. G., \& Thanassoulis, E. (1991). Applied Data Envelopment Analysis, European Journal of Operational Research, 52, 1-15. http://dx.doi.org/10.1016/0377-2217(91)90331-O

Charnes, A., Cooper, W.W., \& Rhodes, E. (1978). Measuring the Efficiency of Decision Making Units, European Journal of Operational Research, 2, 429-444. http://dx.doi.org/10.1016/0377-2217(78)90138-8

Cook, W. D., Kress, M., \& Seiford, L. M. (1992). Prioritization Models for Frontier Decision Making Units in DEA, European Journal of Operational Research, 59, 319-323. http://dx.doi.org/10.1016/0377-2217(92)90148-3

Cooper, W. W., Seiford, L.M., \& Tone, K. (2006). Data Envelopment Analysis : A Comprehensive Text with Models, Applications, References and DEA-Solver Software (2nd ed.), New York, Springer Science. 
Du, J., Liang, L., Chen, Y., Cook, W. D., \& Zhu, J. (2011). A bargaining game model for measuring performance of two-stage network structures, European Journal of Operational Research, $210,390-397$. http://dx.doi.org/10.1016/j.ejor.2010.08.025

Glover, F., \& Sueyoshi, T. (2009). Contributions of Professor William W. Cooper in Operations Research and Management Science, European Journal of Operational Research, 197, 1-16. http://dx.doi.org/10.1016/j.ejor.2008.08.011

Hao, G., Wei, Q., \& Yan, H. (2000). The Generalized DEA Model and the Convex Cone Constrained Game, European Journal of Operational Research, 126, 515-525. http://dx.doi.org/10.1016/S0377-2217(99)00306-9

Kao, C., \& Hung, H. T. (2005). Data envelopment analysis with common weights: the compromise solution approach, Journal of the Operational Research Society, 56, 1196-1203. http://dx.doi.org/10.1057/palgrave.jors.2601924

Nakabayashi, K., \& Tone, K. (2006). Egoist's Dilemma: a DEA Game, Omega, 34, $135-148$. http://dx.doi.org/10.1016/j.omega.2004.08.003

Peters, H. J. M. (1992). Axiomatic Bargaining Game Theory, Kluwer Academic Publishers. http://dx.doi.org/10.1007/978-94-015-8022-9

Roll, Y., Cook, W. D., \& Golany, B. (1991). Controlling Factor Weights in Data Envelopment Analysis, IIE Transactions, 23, 2-9. http://dx.doi.org/10.1080/07408179108963835

Semple, J. (1996). Constrained Games for Evaluating Organizational Performance, European Journal of Operational Research, 96, 103-112. http://dx.doi.org/10.1016/S0377-2217(96)00068-9

Sexton, T. R., Silkman, R. H., \& Hogan, A. J. (1986). Data Envelopment Analysis: Critique and Extensions, Silkman, R. H. (Ed.), Measuring Efficiency: Assessment of Data Envelopment Analysis (pp. 73-105), San Francisco, Jossey Bass.

Statistics Committee of Electric Utilities Association (Ed.). (1992). Hand Book of Electric Power Industry '91 (in Japanese), Tokyo, Japan Electric Association.

Sugiyama, M., \& Yamada, Y. (2000). Data Envelopment Analysis Using Virtual DMU as Intermediates: An Application to Business Analysis of Japan's Automobile Manufatures, Journal of Japan Industrial Management Association, 50, 341-354. http://ci.nii.ac.jp/naid/110003945554/en

Sugiyama, M., \& Yamada, Y. (2001). Group-Consensus Biilding on DEA (in Japanese), Communications of the Operations Research Society of Japan, 46, 284-289. http://ci.nii.ac.jp/naid/110001184921/en

Thomson, W. (1994). Cooperative Models of Bargaining, Aumann, R. J. \& Hart, S. (Eds.), Handbook of Game Theory with Economic Applications (pp. 1237-1284), Elsevier Science B.V..

von Neumann, J., \& Morgenstern, O. (1953). Theory of Games and Economic Behavior (3rd ed.), New Jersey, Princeton University Press.

Yamada, Y., Matsui, T., \& Sugiyama, M. (1994). An Inefficiency Measurement Method for Management Systems (in Japanese), Journal of the Operations Research Society of Japan, 37, 158-168. http://ci.nii.ac.jp/naid/110001184395/en

\section{(cc) $\mathrm{Br}$}

This work is licensed under a Creative Commons Attribution 3.0 License. 